\title{
Application of Massive Transfusion Protocol is Associated with a Low Incidence of Coagulopathy and Mortality Rate
}

\author{
Alhossain Khalafallah ${ }^{1,2 *}$, Abdul-Majeed Albarzan ${ }^{2}$, Amit Ganguly ${ }^{1}$, Gerald Bates ${ }^{1,2}$, Fiona Gavin ${ }^{1}$, Kiran DK Ahuja ${ }^{2}$, David Seaton ${ }^{1}$ and Terry Brain \\ ${ }^{1}$ Launceston General Hospital, Launceston, Tasmania, Australia \\ ${ }^{2}$ School of Human Life Sciences, University of Tasmania, Australia
}

\begin{abstract}
Background: Massive haemorrhage and subsequent Massive Transfusion (MT) are associated with serious, often fatal complications including intractable Disseminated Intravascular Coagulation (DIC). Historically undesirable outcomes demonstrate the need to improve the management approach of MT. It is clear that evidence-based guidelines and protocols should be developed with the aim of improving MT-outcome.
\end{abstract}

Methods: We implemented an evidence-based MT-Protocol (MTP) in 2007 and studied all patients (105) from Jan 2008 to Jan 2011 who required MT and were treated with the MTP at our institution. The MTP includes two phases and incorporates a fixed volume of Fresh Frozen Plasma (FFP), cryoprecipitate and platelets in addition to Packed Red Blood Cells (PRBC).

Results: The median age of patients was 57 years (range, 18-86). The male to female ratio was 74:31. Median $\mathrm{Hb}$ was $90 \mathrm{~g} / \mathrm{L}$ (range, 44-110) and platelet count was 190/nl (range, 34-817). Thirteen patients developed mild DIC; 22 moderate DIC and one severe DIC. D-dimer showed average increase of $7.9 \mathrm{mg} / \mathrm{L}$ with a range between $0.6-35 \mathrm{mg} / \mathrm{L}(\mathrm{Normal}<0.5)$. Average INR was 1.97, (range, 1.2-7.2), while average APTT was 36 seconds (range, 22-88s). The mortality rate was $11.4 \%(12 / 105)$ and these were mainly related to underlying trauma rather than DIC with the exception of 1 patient. The average number of transfused PRBC was 15 units (range, 6-42); cryoprecipitate, 20(range, 10-60); platelets, 2(range, 1-7) and FFP, 8(range, 2-20).

Conclusion: Despite serious bleeding secondary to trauma or surgery, the implementation of MTP seems to have reduced the occurrence of severe DIC and was associated with a relatively low mortality-rate. Further studies to confirm these findings are warranted.

Keywords: Massive transfusion protocol; Massive haemorrhage; Trauma; Surgery; Coagulopathy; Outcome

Abbreviations: DIC: Disseminated Intravascular Coagulation; FFP: Fresh Frozen Plasma; FBC: Full Blood Count; GIT: Gastro Intestinal Tract; ISTH: International Society of Thrombosis and Haemostasis; IV: Intra Venous; MT: Massive Transfusion; MTP: Massive Transfusion Protocol; NBA: National Australian Blood Authority; PRBC: Packed Red Blood Cells; APTT: Activated Partial Thromboplastin Time; PT: Prothrombin Time; VTE: Venous Thrombo Embolism

\section{Introduction}

Trauma is a serious global health problem, accounting for approximately 1 out of 10 deaths worldwide [1-3]. Uncontrollable bleeding accounts for up to $40 \%$ of trauma-related deaths and is the leading cause of potentially preventable death in patients with major trauma $[4,5]$. Massive haemorrhage and subsequent Massive Transfusion (MT) are frequent complications of trauma and surgery $[6,7]$. MT is commonly defined as the replacement of one blood volume in a period of 24 hours, with an alternative dynamic definition of MT used in this study, with replacement of $50 \%$ of the total blood volume within four hours, or a rate of blood loss of $150 \mathrm{ml} / \mathrm{h}$ with foreseeable requirement of blood transfusion, which is more relevant in the acute clinical setting [8-10].

Disseminated intravascular coagulation (DIC) is a serious complication of massive haemorrhage and also subsequent MT associated with trauma or surgery [11-13]. DIC carries a considerable mortality rate, and once established it is difficult to reverse $[14,15]$. Furthermore, DIC remains a clinical challenge that may ultimately end with fatal consequences especially if associated with acidosis and hypothermia [16-17]. Therefore, strategies to prevent DIC are highly warranted in the setting of massive haemorrhage [18-19].

At a single institution, we implemented a Massive Transfusion Protocol (MTP) in late 2007 and studied all patients (105) from Jan 2008 to Jan 2011 who required a MT. MT for our purpose is defined as transfusion of one whole blood volume within 24 hours or replacement of half blood volume within 4 hours with an ongoing need for transfusion.

\section{Aims and Objectives}

The main objective of the MTP is to standardise the approach to treatment and provide appropriate component therapy with the aim of reducing the effect and sequelae of massive haemorrhage, such as exsanguination, coagulopathy and thrombocytopenia. This study was conducted to identify whether a consistent approach with particular attention to the replacement of specific haemostatic blood factors in a timely fashion has an influence on patient outcome and/or reduces DIC.

*Corresponding author: A. Khalafallah, Consultant Haematologist, The Launceston General Hospital, Launceston Tasmania, 7250, Australia, Tel: +61-363487111; Fax: +61-3-63353388; E-mail: khalafallah@dhhs.tas.gov.au

Received March 19, 2012; Accepted May 07, 2012; Published May 13, 2012

Citation: Khalafallah A, Albarzan AM, Ganguly A, Bates G, Gavin F, et al. (2012) Application of Massive Transfusion Protocol is Associated with Low Incidence of Coagulopathy and Mortality Rate. J Blood Disord Transfus 3:123. doi:10.4172/2155-9864.1000123

Copyright: (c) 2012 Khalafallah A, et al. This is an open-access article distributed under the terms of the Creative Commons Attribution License, which permits unrestricted use, distribution, and reproduction in any medium, provided the original author and source are credited. 
Citation: Khalafallah A, Albarzan AM, Ganguly A, Bates G, Gavin F, et al. (2012) Application of Massive Transfusion Protocol is Associated with Low Incidence of Coagulopathy and Mortality Rate. J Blood Disord Transfus 3:123. doi:10.4172/2155-9864.1000123

\section{Patients and Methods}

We studied all 105 patients who presented to the Launceston General Hospital (LGH), the major public tertiary referral hospital in Northern Tasmania, Australia during the period from January 2008 to January 2011. This study has been approved by the Tasmanian Human Research Ethics Committee, Australia. The study is registered with the Australia and New Zealand Clinical Trial Registry (ANZCTR) under trial No: ACTRN12611001258943. Web address of the trial: http:// www.ANZCTR.org.au/ACTRN12611001258943.aspx.

Patients with massive bleeding who required replacement of one whole blood volume within 24 hours or half blood volume within 4 hours with a predictable requirement of ongoing blood transfusion, who presented to the LGH from the Emergency Department or the surgical theatre, were included in this study. These patients had clinical conditions associated with uncontrolled bleeding mainly due to trauma, surgery or invasive procedure.

All patients were tested, as prescribed in the MTP, for Full Blood Count (FBC) and coagulation parameters including platelet count, Prothrombin Time (PT), Activated Partial Thromboplastin Time (APTT), fibrinogen level, and D-dimer. All tests were performed in the Pathology Department at the LGH, a National Australian Testing Authority (NATA)-accredited laboratory. All specimens were analysed within $30 \mathrm{~min}$ of collection. Coagulation assays were performed using a Sysmex CA 1500 analyser and FBCs were performed on a Beckman Coulter LH-500.

The implemented MTP is an evidence-based protocol established in July 2007 at the LGH and was extended to serve the North of Tasmania. It is usually activated by the haematologist or transfusion scientist, and occasionally by anaesthetists, surgeons or ICU/ emergency physicians. The MTP consists of 2 phases as follows: Phase I Protocol, which consists of 4 units packed red cells +2 units Fresh Frozen Plasma (FFP) $(300 \mathrm{ml}$ each $)+1$ adult dose of platelets. This was alternating with Phase II protocol, which consists of 4 units of packed red blood cells +2 units of fresh frozen plasma ( $300 \mathrm{ml}$ each $)+10$ units of cryoprecipitate. The protocol aims for a platelet count $>100 / \mathrm{nL}$, APTT $<45 \mathrm{~s}, \mathrm{PT}<15 \mathrm{~s}$, INR $<1.5$, fibrinogen $>1 \mathrm{~g} / \mathrm{L}$ and $\mathrm{Hb}>70 \mathrm{~g} / \mathrm{L}$ if possible to maintain the haemostasis and minimise the degree of DIC.

Additional blood products may be added or removed from the protocol as required according to the diagnostic results. The protocol prescribes regular FBC, electrolytes and coagulation profiles (including fibrinogen) every 4-6 hours or earlier depending on the severity of the condition and degree of bleeding. Once activated, the MTP remains in force for 24 hours after the last transfusion, unless the treating clinician or haematologist requests prolongation or cessation of the protocol.

\section{DIC measures}

Patients were classified as having or not having DIC according to the International Society of Thrombosis and Haemostasis (ISTH) score [20].

This included occurrence of low platelet count (platelet count $>100 / \mathrm{nL}=0 ;<100 / \mathrm{nL}=1 ;<50 / \mathrm{nL}=2$ ), prolonged prothrombin time $(\mathrm{PT}<3 \mathrm{~s}=0 ;>3$ but $<6 \mathrm{~s}=1 ;>6 \mathrm{~s}=2)$, low fibrinogen level $(>1.0 \mathrm{~g} / \mathrm{L}=0 ;<1.0 \mathrm{~g} / \mathrm{L}=1)$ and raised D-dimer level (no increase = 0 ; moderate increase $=2$; strong increase $=3$ ). Accordingly, the ISTH score was calculated and interpreted as if the overall score was $\geq 5$, it would be compatible with overt DIC [20]. The score was repeated daily until improvement or patient discharge. If the score was $<5$, it would be suggestive (not affirmative) for non-overt DIC. Therefore, the score was repeated in the subsequent $1-2$ days. If the score continues to be $<5$ it is most likely not diagnostic for DIC [20].

\section{Statistical methods}

Data were analysed using mixed methods linear regression (STATA software, version 12SE, Stata corp. Texas, USA). Assumptions of linear regression were checked and if violated data were analysed using ordinal logistic regression. Post hoc (Holm's test) was used to calculate $\mathrm{p}$ values for repeated measures. P-value $<0.05$ was considered as statistically significant.

\section{Results}

Of the 105 patients investigated, 49 patients were the subject of trauma and 45 were surgical patients, which included surgery performed to control bleeding. The cohort also included patients who bled during standard invasive surgical procedures (5 cases) or were on warfarin therapy (6 cases). The surgery subtypes are demonstrated in (Table 1).

The median age of patients was 57 years (range 18-86) with male to female ratio of (74:31). Median $\mathrm{Hb}$ was $90 \mathrm{~g} / \mathrm{L}$ (range 44110) with a median platelet count of $190 / \mathrm{nl}$ (range 34-817). DIC was present to varying degrees from mild to moderate. D-dimer showed average increase of $7.9 \mathrm{mg} / \mathrm{L}$ ( Normal $<0.5$ ) with a range between 0.6 35.1. Average INR was 1.97 with a range between 1.2-7.2 $(\mathrm{N} .<1.1)$ while average APTT was $36 \mathrm{~s}$ with a range of 22-88s (N. 15-34s). The mortality rate was $11.4 \%(12 / 105)$ and was mainly related to the presenting trauma or surgery rather than DIC with the exception of one patient (Figure 1). The average number of RBC units transfused was 15 (range, 6-42); platelets, two units (range 1-7); cryoprecipitate, 20 units (range, 10-60); while the number of FFP units was 8 units (range, 2-20). Recombinant factor VIIa (Novoseven ${ }^{\circ}$, NovoNordisk) was administered to one patient. Intravenous (IV) prothrombin concentrate (Prothrombinex) in doses ranging from 1000 to $3000 \mathrm{IU}$

\begin{tabular}{|l|l|}
\hline Number of patients & 105 \\
\hline Age median years & $\begin{array}{l}57 \text { years (range 18 } \\
\text { to 86). }\end{array}$ \\
\hline Male to female ratio & $74: 31$ \\
\hline No of Trauma patients & 49 \\
No of Surgical patients & 45 \\
No of patients who bled as complication of surgery & 35 \\
No of patients who required surgery to cease bleeding & 10 \\
No of patients who massively bled associated with warfarin & 6 \\
No of patients who underwent invasive procedures & 5 \\
\hline Type of surgery & \\
GIT surgery & 12 \\
Genitourinary tract surgery & 5 \\
Orthopaedic surgery & 9 \\
Obstetrics and Gynaecology & 7 \\
Vascular Surgery & 5 \\
General surgery & 8 \\
\hline Laboratory coagulation parameters & Average (range) \\
D-dimer (Normal <0.5 mg/L) & 7.9 mg/L (0.6-35) \\
INR (N. <1.1) & $1.97(1.2-7.2)$ \\
APTT (N. 23-34 s) & 36 s (22-88s) \\
\hline Blood products: & \\
Average No of cryoprecipitate units & 20 (range) (10-60) \\
Average No of FFP units & 8 (range) (2-20) \\
Average No of adult platelets units & 2 (range) (1-7) \\
Average No of PRBC units & 15 (range) (6-42) \\
\hline
\end{tabular}

GIT: Gastrointestinal tract; FFP: Fresh frozen plasma; PRBC: Packed red blood cells

Table 1: Cohort statistics. 
Citation: Khalafallah A, Albarzan AM, Ganguly A, Bates G, Gavin F, et al. (2012) Application of Massive Transfusion Protocol is Associated with Low Incidence of Coagulopathy and Mortality Rate. J Blood Disord Transfus 3:123. doi:10.4172/2155-9864.1000123

Page 3 of 5

was administered to 13 patients with prolonged PT/INR, of whom 6 were on warfarin therapy at the time.

Twelve patients were on aspirin therapy and one patient was on clopidogrel. Moderate DIC was present in 22 patients (INR between 1.5-2 without warfarin) and mild DIC (INR 1.1-1.5) was present in 13 patients, while one patient developed severe DIC with ischaemic gut and succumbed thereafter.

Of those patients who died, the attributed causes of death were Staphylococcus aureus sepsis ( 1 case), GIT surgery with additional respiratory failure ( 2 cases), hepatic failure and renal failure ( 2 cases), severe multi-trauma injury ( 2 cases), cardiopulmonary shock (1 case), multi-organ failure (2 cases), ischaemic gut, severe DIC and profound

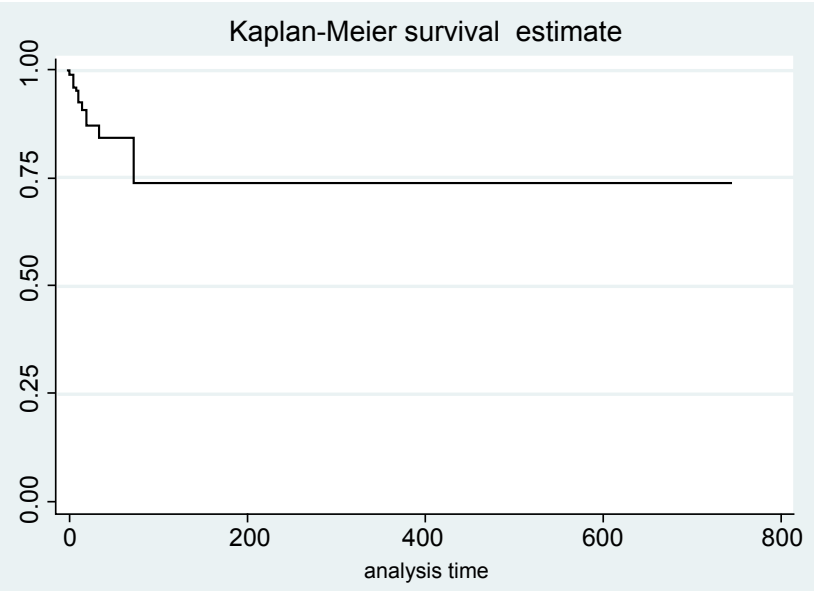

Figure 1: All cause mortality in studied patients who received Massive Transfusion Protocol at a single centre.

\begin{tabular}{|l|c|}
\hline Cause of death & No of cases \\
\hline Hepatic and renal failure & 2 \\
\hline Cardiopulmonary shock & 2 \\
\hline Multi-organ failure including respiratory & 2 \\
\hline Severe gut ischaemia with intractable DIC & 1 \\
\hline Severe multi-trauma injury & 2 \\
\hline GIT-surgery including an oncology case & 2 \\
\hline Staph Aureus sepsis & 1 \\
\hline
\end{tabular}

GIT: Gastrointestinal Tract; DIC: Disseminated Intravascular Coagulation

Table 2: Causes of mortality.

\begin{tabular}{|c|c|c|c|c|}
\hline Parameter & $\begin{array}{l}\text { Initial Mean } \\
\text { (SD) }\end{array}$ & $\begin{array}{c}\text { Post transfusion } \\
\text { Mean (SD) }\end{array}$ & Exit Mean (SD) & $\begin{array}{c}\text { Overall } \\
\text { p-value } \\
\text { (holms test) }\end{array}$ \\
\hline Haemoglobin & $100.27(28.68)$ & $\begin{array}{l}83.99(18.47) \\
P \text { value against } \\
\text { initial }<0.001\end{array}$ & $\begin{array}{l}96.19(13.90) \\
\text { P value against } \\
\text { post transfu- } \\
\text { sion }=\mathbf{0 . 0 0 7}\end{array}$ & $<0.001$ \\
\hline Platelets & $\begin{array}{l}267.16 \\
(164.40)\end{array}$ & $\begin{array}{l}173.37(132.29) \\
P \text { against initial } \\
<0.001\end{array}$ & $\begin{array}{l}143.2(80.40) \\
P \text { against trans- } \\
\text { fusion }<0.001\end{array}$ & $<0.001$ \\
\hline HCT & $0.33(0.31)$ & $0.28(0.05) \mathrm{NS}$ & $0.35(0.45) \mathrm{NS}$ & 0.45 \\
\hline INR & $1.74(1.55)$ & 1.67 (1.19) NS & $1.97(4.13) \mathrm{NS}$ & 0.81 \\
\hline APTT & $36.13(15.13)$ & $\begin{array}{l}39.85(21.05) \\
\text { P against } \\
\text { initial= } \mathbf{0 . 0 9}\end{array}$ & $\begin{array}{l}32.87(16.57) \\
P \text { against trans- } \\
\text { fusion= } \mathbf{0 . 0 2}\end{array}$ & 0.02 \\
\hline
\end{tabular}

SD: Standard Deviation; HCT: Haematocrit; NS: No Significant Difference

Table 3: Significance of diagnostic results at different time points. circulatory shock ( 1 case) as shown in (Table 2). There were 6 patients who succumbed in the trauma group $(12.2 \%)$ vs. 6 patients in the surgical procedures group (10.7\%). Further analysis of the amount of transfused units of blood in correlation with mortality showed that among 28 patients who had 6-16 units of blood, there was $1.9 \%$ mortality compared to $3.8 \%$ in 41 patients who received between $17-24$ units of blood. There was $5.7 \%$ mortality in 36 patients who had $>24$ units.

A comparison of the diagnostic values of $\mathrm{Hb}$, platelets and coagulation parameters at the different time points is demonstrated in (Table 3). These diagnostic parameters, which usually correlate with DIC, showed significant improvement on the average from the time of admission through the treatment period and prior to discharge. The APTT level showed a significant improvement at time of discharge ( $\mathrm{p}=0.02$ ), while PT/INR levels did not change significantly, possibly indicating a longer recovery time. The platelet and $\mathrm{Hb}$ levels were improved significantly by discharge $(\mathrm{p}<0.001)$ (Table 3$)$. Unfortunately, follow-up coagulation testing was not performed routinely after discharge and was therefore not available for comparison. Furthermore, we did not notice any correlation between different phases of the protocol and occurrence of DIC and we did not record any thrombotic complications in our cohort of patients.

\section{Discussion}

Our data show that the application of MTP is useful in a medical centre that is involved in retrieval services and actively manages trauma patients, or when uncontrolled bleeding after high-risk surgery is anticipated.

A noted common clinical practice in these situations is that the treating clinician will often focus on restoring blood volume and in maintaining blood pressure as their first life-saving measure [21]. However, MTP serves to add focus to the haemostasis as well as restoring blood volume and pressure in this context, hence improving the outcome.

The debate remains over which product should or could be used in the setting of acute blood loss, whether fresh whole blood is superior to component therapy. However, the reality is that resourcing demands dictate that availability in Australia and most other parts of the world is restricted to the component options with very few centres able to access and utilise fresh whole blood [21-23].

Protocols developed for component therapy generally apply proportionate Packed Red Blood Cells (PRBC) to Fresh Frozen Plasma (FFP). Some uncertainty remains over the appropriate proportions [23-24]. FFP contains only small amounts of fibrinogen, an important factor in management of DIC and uncontrolled bleeding [24-25]. Therefore, our MTP incorporates fixed proportional amounts of PRBC and FFP as well as cryoprecipitate with the latter as a rich source of fibrinogen [22-27]. Despite the use of different coagulation factors, we did not record any Venous Thrombo Embolic (VTE) complications. This is mainly due to the following factors; 1) We administered mainly FFP and cryoprecipitate that are considered as having diluted coagulation factors and hence, less likelihood of VTE complications 2) The use of prothrombin concentrate in this protocol was mainly to reverse vitamin-K antagonist (warfarin) and hence, was associated with low occurrence of VTE. 3) Recombinant FVIIa is a known risk of VTE, however it was used only in 1 patient who did have severe bleeding and did not develop any thrombotic complications thereafter.

Although some studies suggest that treatment of trauma patients 
with a certain ratio of $\geq 1: 1$ of FFP: RBC significantly reduced the intra-operative, 1-day and 30-day mortality [28-30], there is a paucity of randomized prospective trials in this cohort of patients addressing the optimal FFP:RBC ratio owing to the many technical and logistic difficulties. However, many studies support early and aggressive replacement of plasma coagulation factors [31-34]. Another crucial factor is the timing of when to apply FFP. It has been reported that a significant reduction in mortality, from $45 \%$ to $19 \%$, after implementation of an MTP despite unchanged FFP: RBC ratios, is most likely due to a reduction of the average time to administer FFP, from 4.5 to 3 hours [35]. Therefore, several studies conclude that early haemostatic-support seems to reduce coagulopathy in some patients and sometimes eliminates the need for MT at all [28-35]. Furthermore, some studies suggest that FFP is the most effective component in avoiding DIC when applied during the first 2-3 hours of MTP [36].

In contrast to a fixed ratio of coagulation products to RBCs, target-directed coagulation components focus on actual needs of the individual patient, based on coagulation results $[5,37,38]$. However the time-lag of the turnaround times of different coagulation tests stands as an obstacle to individualized coagulation component replacement in a timely fashion and perhaps contributes to adverse outcomes in such situations.

In our hands, we have a lower incidence of coagulopathy and mortality rate due to coagulopathy $(<1 \%)$ compared to other published series [4,14]. In an audit of the German Trauma Registry of 17,200 multiple injured patients, $29 \%$ of patients with coagulopathy developed multi organ failure $(\mathrm{p}<0.001)$ and the early mortality $(<24 \mathrm{~h})$ among this cohort of patients was $13 \%(p<0.001)$ [14]. The same study documented an overall in-hospital mortality of $28 \%(\mathrm{p}<0.001)$ for all trauma patients included in the report [14].

Nevertheless, it is not only important to increase the awareness and necessity of implementing a MTP, but also to recognise and address the need for clinicians, transfusion services, blood banks and laboratories to support and maintain the sustained application of MTP $[26,27]$. Some of the factors that may influence the outcome of MT are considered centre or hospital specific; such as arrangements with the laboratory, blood product availability, use of emergency-pack for blood transfusion, in addition to in-house policies. These factors must be given due consideration when applying a MTP. Nonetheless, early correction of coagulopathy remains the hallmark that determines the success of such protocols.

Our MTP is well aligned with the recently launched National Australian Blood Authority (NBA) guideline for massive transfusion, which also recommends the development of a local adaptation of protocols that define pathways for treatment of critically ill patients who require massive transfusion support [39]. The NBA-MTP guidelines include two units of FFP for every issued four units of PRBC, considering 1 adult unit of platelets and also cryoprecipitate when fibrinogen $<1 \mathrm{~g} / \mathrm{L}$ similar to what our protocol suggests. The main difference between our protocol and the NBA protocol is that the latter considering cryoprecipitate and platelets when required, while our MTP is standardizing the ratio of these blood products in case of MT.

Furthermore, it is important to develop a strategy that integrate the key players from different disciplines in order to initiate the most appropriate management of massive haemorrhage in a timely fashion. The strategy should take into account: (1) Emergency O-negative PRBC available for retrieval and emergency situations that is always ready for urgent use. (2) Base-line blood testing of the patients' FBC, coagulation studies, blood group and screen, cross-match, electrolytes must be routinely performed. (3) The time-lag from patient presentation to the availability of blood test result and subsequent action taken by the treating physician with regards to these results. These factors are possibly influencing the MT-outcome and therefore it should be considered and optimized. It is worth noting that there is a lack of data and clinical trials addressing these issues.

The MTP used in this study has attempted to standardise the amount of FFP, cryoprecipitate and platelets, whilst allowing flexibility with the integration of extra blood products based on laboratory parameters. This has successfully reduced the occurrence of DIC and improved the outcome in this group of patients.

The requirement of extra anti-fibrinolytic measurements such as tranexamic acid $(0 \%)$ or the use of pro-coagulants such as activated FVII $(<1 \%)$ or prothrombin concentrate $(7$ cases $)$ in our cohort of patients is considered minimal as we excluded the use of prothrombin concentrate in reversing vitamin $\mathrm{K}$-antagonist (warfarin).

In summary, our study shows a lower coagulopathy and mortality rate (11.4\%) compared to historic data, without incidence of serious or irreversible DIC except for one case $[4,5,14,34,35]$. However, it is worth noting that in our series, we included patients who bled massively after surgical procedures and this may have contributed to the favourable outcome of our MTP. The mortality rate among trauma patients (12.2\%) was higher than mortality rate secondary to massive bleeding after surgical procedures (10.7\%). Our study has a few shortcomings; firstly; it includes a heterogeneous group of patients who required MTP. Secondly; it is an audit of a current MTP and is not a randomized trial because of the inherent difficulties of recruiting and randomizing the targeted acute critically ill patients. Therefore we used historic data as a point of reference.

Altogether, MTP may be useful in the setting of acute blood loss in different clinical scenarios for the avoidance of DIC and hence easing microbleeding as early as possible with the consequence of improving outcomes. Therefore, in order to maximize the benefits and effectiveness of such MTPs, refinement will be achieved by adapting local national blood transfusion guidelines and incorporating local health service area requirements that are warranted for the successful implementation of an MTP.

In conclusion, our data show that early intervention with haemostatic support is associated with a low incidence of severe DIC and hence, lower mortality rate. Further studies to confirm this data are warranted.

\section{Acknowledgements}

The authors would like to thank sincerely the laboratory staff at the Launceston General Hospital Pathology, who provided the information for this review and the blood products to the patients included in the study.

\section{References}

1. http://www.who.int/mediacentre/news/releases/2004/pr24/en/index.html

2. Cothren CC, Moore EE, Hedegaard HB, Meng K (2007) Epidemiology of urban trauma deaths: a comprehensive reassessment 10 years later. World J Surg 31: 1507-1511.

3. Hofman K, Primack A, Keusch G, Hrynkow S (2005) Addressing the growing burden of trauma and injury in low-and middle-income countries. Am J Public Health 95: 13-17

4. Kauvar DS, Lefering R, Wade CE (2006) Impact of hemorrhage on trauma outcome: an overview of epidemiology, clinical presentations, and therapeutic considerations. J Trauma 60: S3-11. 
Citation: Khalafallah A, Albarzan AM, Ganguly A, Bates G, Gavin F, et al. (2012) Application of Massive Transfusion Protocol is Associated with Low Incidence of Coagulopathy and Mortality Rate. J Blood Disord Transfus 3:123. doi:10.4172/2155-9864.1000123

Page 5 of 5

5. Schöchl H, Maegele M, Solomon C, Görlinger K, Voelcke W (2012) Early and individualized goal-directed therapy for trauma-induced coagulopathy. Scand $\mathrm{J}$ Trauma Resusc Emerg Med 20: 15.

6. Hess JR, Zimrin AB (2005) Massive blood transfusion for trauma. Curr Opin Hematol 12: 488-492

7. Cotton BA, Au BK, Nunez TC, Gunter OL, Robertson AM, et al. (2009) Predefined massive transfusion protocols are associated with a reduction in organ failure and postinjury complications. J Trauma 66: 41-48.

8. Mannucci PM, Levi M (2007) Prevention and treatment of major blood loss. N Engl J Med 356: 2301-2311.

9. Murthi SB, Stansbury LG, Dutton RP, Edelman BB, Scalea TM, et al. (2011) Transfusion medicine in trauma patients: an update. Expert Rev Hematol 4 : 527-537.

10. Perkins JG, Cap AP, Weiss BM, Reid TJ, Bolan CD (2008) Massive transfusion and nonsurgical hemostatic agents. Crit Care Med 36: S325-S339.

11. Brohi K, Singh J, Heron M, Coats T (2003) Acute traumatic coagulopathy. J Trauma 54:1127-1130.

12. Levi $M$, Fries $D$, Gombotz $H$, van der Linden $P$, Nascimento $B$, et al. (2011) Prevention and treatment of coagulopathy in patients receiving massive transfusions. Vox Sang 101: 154-174.

13. Niles SE, McLaughlin DF, Perkins JG, Wade CE, Li YZ, et al. (2008) Increased mortality associated with the early coagulopathy of trauma in combat casualties. J Trauma 64: 1459-1463.

14. Maegele M, Lefering R, Yucel N, Tjardes T, Rixen D, et al. (2007) Early coagulopathy in multiple injury: an analysis from the German Trauma Registry on 8724 patients. Injury 38: 298-304.

15. Hardy JF, de Moerloose P, Samama CM; Members of the Groupe d'Intérêt en Hémostase Périopératoire (2006) Massive transfusion and coagulopathy: pathophysiology and implications for clinical management. Can J Anaesth 53: S40-S58.

16. Sihler KC, Napolitano LM (2010) Complications of massive transfusion. Chest 137: 209-220.

17. Cosgriff N, Moore EE, Sauaia A, Kenny-Moynihan M, Burch JM, et al. (1997) Predicting life-threatening coagulopathy in the massively transfused trauma patient: hypothermia and acidoses revisited. J Trauma 42: 857-861.

18. Shaz BH, Dente CJ, Harris RS, MacLeod JB, Hillyer CD (2009) Transfusion management of trauma patients. Anesth Analg 108: 1760-1768

19. Rossaint R, Bouillon B, Cerny V, Coats TJ, Duranteau J, et al. (2010) Management of bleeding following major trauma: an updated European guideline. Crit Care 14: R52.

20. Toh $\mathrm{CH}$, Hoots WK; SSC on Disseminated Intravascular Coagulation of the ISTH (2007) The scoring system of the Scientific and Standardisation Committee on Disseminated Intravascular Coagulation of the International Society on Thrombosis and Haemostasis: a 5-year overview. J Thromb Haemost 5: 604-606.

21. Arnold JL, Dickinson G, Tsai MC, Han D (2001) A survey of emergency medicine in 36 countries. CJEM 3: 109-118.

22. Gould S, Cimino MJ, Gerber DR (2007) Packed red blood cell transfusion in the intensive care unit: limitations and consequences. Am J Crit Care 16: 39-48.

23. Lynn M, Jeroukhimov I, Klein Y, Martinowitz U (2002) Updates in the management of severe coagulopathy in trauma patients. Intensive Care Med 28: $241-247$.

24. Gonzalez EA, Moore FA, Holcomb JB, Miller CC, Kozar RA, et al. (2007) Fresh frozen plasma should be given earlier to patients requiring massive transfusion. J Trauma 62: 112-119

25. Stinger HK, Spinella PC, Perkins JG, Grathwohl KW, Salinas J, et al. (2008) The ratio of fibrinogen to red cells transfused affects survival in casualties receiving massive transfusions at an army combat support hospital. J Trauma 64: S79-S85.

26. Erber WN (2002) Massive blood transfusion in the elective surgical setting Transfus Apher Sci 27: 83-92.

27. Malone DL, Hess JR, Fingerhut A (2006) Massive transfusion practices around the globe and a suggestion for a common massive transfusion protocol. $J$ Trauma 60: S91-S96.

28. Borgman MA, Spinella PC, Perkins JG, Grathwohl KW, Repine T, et al. (2007) The ratio of blood products transfused affects mortality in patients receiving massive transfusions at a combat support hospital. J Trauma 63: 805-813.

29. Holcomb JB, Wade CE, Michalek JE, Chisholm GB, Zarzabal LA, et al. (2008) Increased plasma and platelet to red blood cell ratios improves outcome in 466 massively transfused civilian trauma patients. Ann Surg 248: 447-458.

30. Lustenberger T, Frischknecht A, Bruesch M, Keel MJ (2011) Blood component ratios in massively transfused, blunt trauma patients--a time-dependent covariate analysis. J Trauma 71: 1144-1150.

31. Maegele M, Lefering R, Paffrath T, Tjardes T, Simanski C, et al. (2008) Red-blood cell to plasma ratios transfused during massive transfusion are associated with mortality in severe multiple injury: a retrospective analysis from the Trauma Registry of the Deutsche Gesellschaft fur Unfallchirurgie. Vox Sang 95: $112-119$

32. Murad MH, Stubbs JR, Gandhi MJ, Wang AT, Paul A, et al. (2010) The effect of plasma transfusion on morbidity and mortality: a systematic review and metaanalysis. Transfusion 50: 1370-1383.

33. Davenport R, Curry N, Manson J, De'Ath H, Coates A, et al. (2011) Hemostatic effects of fresh frozen plasma may be maximal at red cell ratios of $1: 2$. J Trauma 70: 90-95.

34. Dirks J, Jorgensen H, Jensen CH, Ostrowski SR, Johansson PI (2010) Blood product ratio in acute traumatic coagulopathy--effect on mortality in a Scandinavian level 1 trauma centre. Scand J Trauma Resusc Emerg Med 18: 65.

35. Riskin DJ, Tsai TC, Riskin L, Hernandez-Boussard T, Purtill M, et al. (2009) Massive transfusion protocols: the role of aggressive resuscitation versus product ratio in mortality reduction. J Am Coll Surg 209: 198-205.

36. de Biasi AR, Stansbury LG, Dutton RP, Stein DM, Scalea TM, et al. (2011) Blood product use in trauma resuscitation: plasma deficit versus plasma ratio as predictors of mortality in trauma (CME). Transfusion 51: 1925-1932.

37. Johansson PI (2010) Goal-directed hemostatic resuscitation for massively bleeding patients: the Copenhagen concept. Transfus Apher Sci 43: 401-405.

38. Kashuk JL, Moore EE, Johnson JL, Haenel J, Wilson M, et al. (2008) Postinjury life threatening coagulopathy: is $1: 1$ fresh frozen plasma:packed red blood cells the answer? J Trauma 65: 261-270.

39. http://www.nba.gov.au/guidelines/module1/index.html 